\title{
Presentation and outcome of subsequent thyroid cancer among childhood cancer survivors compared to sporadic thyroid cancer: a matched national study
}

\author{
Sarah C Clement ${ }^{1,2}$, Chantal A Lebbink 1,4, Mariëlle S Klein Hesselink ${ }^{3}$, Jop C Teepen ${ }^{4}$, Thera P Links ${ }^{3}$, \\ Cecile M Ronckers ${ }^{4,5}$ and Hanneke M van Santen ${ }^{1,4}$ on behalf of the DCOG-LATER Study Group, The Netherlands \\ and the Dutch (Pediatric) Thyroid Cancer Consortium
}

${ }^{1}$ Department of Pediatric Endocrinology, Wilhelmina Children's Hospital/University Medical Center Utrecht, Utrecht, The Netherlands, ${ }^{2}$ Department of Pediatrics, Amsterdam University Medical Center location VU Medical Center, Amsterdam, The Netherlands, ${ }^{3}$ Department of Endocrinology, University Medical Center Groningen, University of Groningen,

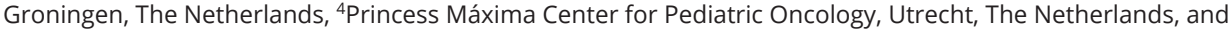
${ }^{5}$ Medical University Brandenburg - Theodor Fontane, Institute of Biostatistics and Registry Research, Neuruppin, Germany

Correspondence should be addressed to $\mathrm{H} \mathrm{M}$ van Santen Email

h.m.vansanten@ umcutrecht.nl

\begin{abstract}
Objective: Childhood cancer survivors (CCS) are at increased risk to develop differentiated thyroid cancer predominantly after radiotherapy (subsequent DTC). It is insufficiently known whether subsequent DTC in CCS has a different presentation or outcome than sporadic DTC.

Methods: Patients with subsequent DTC $(n=31)$ were matched to patients with sporadic DTC $(n=93)$ on gender, age and year of diagnosis to compare presentation and DTC outcomes. Clinical data were collected retrospectively. Results: Among the CCS with subsequent DTC, all but one had received chemotherapy for their childhood cancer, $19(61.3 \%)$ had received radiotherapy including the thyroid region, $3(9.7 \%){ }^{131}$ I-MIBG and 8 (25.8\%) had received treatment with chemotherapy only. Subsequent DTC was detected by surveillance through neck palpation (46.2\%), as a self-identified mass (34.6\%), or by chance. Among sporadic DTC patients, self detection predominated (68.8\%). CCS with subsequent DTC tended to have on average smaller tumors (1.9 vs $2.4 \mathrm{~cm}$, respectively, $(P=0.051)$, and more often bilateral $(5 / 25(60.0 \%)$ vs $28 / 92(30.4 \%), P=0.024)$. There were no significant differences in the occurrence of surgical complications, recurrence rate or disease-related death.

Conclusion: When compared to patients with sporadic DTC, CCS with subsequent DTC seem to present with smaller tumors and more frequent bilateral tumors. Treatment outcome seems to be similar. The finding that one-third of subsequent DTC cases had been treated with chemotherapy only needs further investigation. These results are important for the development of surveillance programs for CCS at risk for DTC and for treatment guidelines of subsequent DTC.

\section{Introduction}

Treatment of pediatric malignancies has improved substantially over the past several decades, resulting in a rapidly growing population of long-term childhood cancer survivors (CCS) (1). CCS are at a risk to develop subsequent malignancies, of which approximately $10 \%$ involve the thyroid gland $(2,3,4,5)$. 
The occurrence of differentiated thyroid carcinoma (DTC) in CCS is predominantly attributable to radiation therapy that directly or incidentally involves the thyroid gland (6). The risk for subsequent DTC increases linearly with increasing estimated dose to the thyroid gland, with a plateau around 10-30 Gy and a decline at higher dose $(2,6)$. Ionizing radiation exposure at a young age (e.g. $<5$ years) confers an additional risk factor for DTC after radiotherapy (6). The occurrence of DTC has also been reported among survivors of neuroblastoma treated with ${ }^{131}$ I-Metaiodobenzylguanidine (MIBG) (7). Moreover, in recent years, a possible role of chemotherapeutic agents in the etiology of DTC is emerging, consistent with evidence for other subsequent malignancies $(5,6,8)$.

Children with DTC generally have excellent survival rates, even if the disease presents at a more advanced stage (9). The Dutch Childhood Oncology Group (DCOGLATER) recommends yearly physical examination (palpation) of the thyroid gland for CCS at increased risk for DTC based on previously given treatments (10). Screening for DTC in CCS can also be done using thyroid ultrasound. In a recent report of the International Guideline Harmanization Group (IGHG) the pros and cons of both surveillance strategies were summarized, and it was recommended to counsel the survivor and, using shared-decision-making, determine the optimal mode of surveillance for each specific individual (11). Treatment for DTC is currently performed in accordance with established treatment algorithms for sporadic thyroid cancer $(12,13)$.

There are several studies that report generally a similar clinical presentation and outcome of DTC among ionizing radiation-exposed patients in comparison to individuals with sporadic DTC $(14,15,16,17,18,19,20,21,22,23$, $24)$. These reports, however, concerned predominantly unmatched studies focusing on patients who received radiotherapy for benign lesions or patients who were exposed to radioiodine isotopes released by nuclear reactors. To strengthen the evidence for development of appropriate surveillance and treatment strategies for these individuals, more information is needed in matched cohorts to compare the mode of detection and presentation of disease as well as the outcome.

For these reasons, the aim of the present study was to evaluate the mode of detection, presentation, treatment and outcome of subsequent DTC among CCS in The Netherlands in comparison with a matched sample of patients with sporadic DTC. If such differences were to be found, it could be used to inform current care practices (screening and management) in CCS. To address our objectives, we conducted retrospective chart review studies based on several nationwide patient cohorts.

\section{Subjects and methods}

\section{Study populations}

We included two source populations to identify CCS with subsequent DTC: (1.) The DCOG-LATER cohort of more than 60005 -year CCS diagnosed with a primary tumor between 1963 and 2001 in The Netherlands (5). Clinical follow-up and record linkage studies (including cancer and pathology registries), with follow-up coverage until January 1, 2013, revealed 28 patients with subsequent DTC (5). To complete the inclusion of subsequent DTC patients (period 2013-2015), additional record linkage with the nationwide network and registry of histo- and cyto-pathology in The Netherlands (PALGA) revealed two additional cases with subsequent DTC (25); (2.) To obtain subsequent DTC cases among CCS initially diagnosed after 2001, we queried The Netherlands Cancer Registry (IKNL) and identified one additional eligible case. Of these 31 DTC patients, one patient had DTC as third malignancy. The first and subsequent malignancies among CCS were histologically confirmed.

Every subsequent DTC patient was matched to three sporadic DTC patients (total comparison group $n=93$ ), based on gender, age at DTC diagnosis ( \pm 2 years), and year of DTC diagnosis ( \pm 5 years). These strict criteria were met for $24 / 31$ subsequent DTC patients. In four cases it was not possible to match for calendar years, and in three cases we were not able to match for gender. These subsequent DTC patients were matched with the best possible matching sporadic DTC patient. Eligible sporadic DTC patients were sampled from two patient series using the following inclusion criteria: (1.) diagnosed with a primary DTC, that is, papillary thyroid carcinoma, papillary microcarcinoma $(<1 \mathrm{~cm})$, or follicular carcinoma; (2.) no history of another malignancy; (3.) no exposure to radiotherapy, chemotherapy or hematopoietic stem cell transplantation for non-thyroid neoplasia or benign lesions. For CCS diagnosed with subsequent DTC during childhood $(\leq 18$ years) $(n=9)$, matching subjects $(n=27)$ with sporadic DTC were identified from an ongoing National Pediatric DTC study (26). For CCS who developed subsequent DTC after the age of $18(n=22)$, matching was done with subjects of the adult study cohort with sporadic DTC from the University Medical Center of Groningen $(n=66)(27)$. 
No further approval for retrospective data collection and analysis was needed, in accordance with Dutch law.

\section{Data collection and study definitions}

Data were collected retrospectively and extracted from existing databases $(5,26,27)$ or collected from individual patients' medical records. Pediatric cancer treatment histories for subsequent DTC patients were taken from the DCOG-LATER registry. Radiotherapy exposing the thyroid gland included the following fields: neck (cervical+mantle), head/brain, mediastinum, or total body irradiation (TBI). Cumulative thyroid-directed dose was based on the prescribed radiotherapy dose to the smallest field of the neck; full-field and boost dose were summed.

For all patients, cytology and histopathology findings were scored to determine the characteristics of DTC. Fine needle aspiration cytology (FNAC) results recorded within 6 months of a histological DTC diagnosis were used to define a confirmed DTC. Non-diagnostic findings were, in general, cytological smear samples with too little cells to allow a diagnosis. Diagnostic findings were subdivided into: malignant findings, indeterminate findings, and benign findings. Histopathological DTC data were obtained from the original pathology reports and reviewed by two reviewers (CL/HvS).

Tumor staging was recorded according to the 7th edition TNM (Tumor, Node, Metastasis) classification system for DTC of the Union for International Cancer Control (UICC). TNM stage was classified after the first I-131 treatment. If previous editions were used at time of diagnosis, tumor stage was reclassified into the 7 th edition (28).

All patients had been treated for DTC in accordance with local protocols. Initial neck dissection was defined as those performed at most 1 year after initial thyroid surgery. To calculate the cumulative administered I-131 activity, only ablative and therapeutic I-131 administrations were taken into account.

Documented transient hypoparathyroidism was defined as postoperative hypocalcaemia (serum calcium value below the reference range) with recovery and no use of medication (Calcitriol, Alfacalcidol or calcium) at 6 months after surgery. Documented permanent hypoparathyroidism was defined as postoperative hypocalcaemia with the use of medication (Calcitriol, Alfacalcidol, or calcium) at the last moment of follow-up. Documented transient and documented permanent recurrent laryngeal nerve (RLN) injury after surgery were defined, respectively, as injury mentioned in ear, nose and throat report, or if no report was available, in other medical records, with or without recovery within 6 months after surgery.

Recurrence of DTC was defined as laboratory or radiological evidence of disease activity after remission. Remission of DTC was defined as clinical, radiological and scintigraphic absence of disease activity with an undetectable serum thyroglobulin (Tg) concentration $(<1.0 \mathrm{ng} / \mathrm{mL})$ at least 1 year after the last I-131 therapy. Persistent disease was defined as persistent disease or recurrence at last moment of follow-up. If no follow-up data were known, the disease status at last moment of follow-up was noted as unknown. Outcome was only assessed if the last treatment for DTC had been given $>1$ year ago.

\section{Statistical analysis}

Baseline factors, cancer-related characteristics, treatment modalities and outcome in patients with subsequent DTC and sporadic DTC were compared using Student's $t$-test for continuous measurements. Mann-Whitney $U$-tests were performed if the continuous data were non-normally distributed. For categorical data chi-square tests or Fisher's exact tests (if the assumptions for chi-square were violated) were used. Tests were only performed if reasonably complete information on the clinical characteristics of interest was obtained, defined as $>50 \%$ of each group. All $P$-values were based on two-sided testing and $P$-values of $<0.05$ were considered as statistically significant. The SPSS (25.0) statistical package was used for analysis.

\section{Results}

\section{Patient characteristics}

CCS with subsequent DTC

Thirty-one eligible CCS with subsequent DTC were included. Median age at diagnosis of the primary childhood cancer was 6.1 (range: 0.3-16.4) years, of which leukemias (35.5\%) and lymphomas (19.4\%) were most frequent. Almost all patients (93.5\%) had received chemotherapy, and alkylating agents had been administered in $75.9 \%$ of the CCS and anthracyclines in 55.2\%. Radiotherapy to a field 1 including the thyroid gland was given to 18 patients $(58.1 \%)$, with a mean cumulative dose of 32.0 (range 7.0-55.8) Gy. Three patients (9.7\%) had received ${ }^{131}$ I-MIBG treatment, with a mean total activity of 12.93 GBq for neuroblastoma, including thyroid prophylaxis 
$(8,29)$ Median latency time between primary childhood cancer and subsequent DTC was 17.2 years (range 5.7-33.8) years.

\section{Comparison with sporadic DTC patients}

Diagnosis of DTC Nearly half of the subsequent DTCs $(12 / 26)$ were detected by neck palpation at the DCOGLATER outpatient clinic and 9/26 detected a mass in the neck themselves. Five out of 26 subsequent DTC were found as chance finding during diagnostic work-up for hypothyroidism, hyperparathyroidism or PET scanning for non-Hodgkin lymphoma follow-up. When compared to sporadic DTC patients, 33/48 detected a thyroid mass themselves which was significantly different.

At DTC diagnosis, palpability of the nodule at physical examination, median tumor size and presence of palpable pathological cervical lymph nodes were all comparable between subsequent and sporadic DTC (Table 1).

Ultrasound, cytology and histology findings Pre-operative ultrasound reports were collected and could be retrieved in $58.1 \%$ of CCS and in $15.1 \%$ of the matched patients. Maximum nodule size on ultrasound did not differ between subsequent and sporadic DTC patients (mean 2.3 ( \pm S.D. 1.2) and 2.6 ( \pm S.D. 0.9) cm, respectively).

Overall distribution of FNAC results (within 6 months of a histological DTC diagnosis) were comparable (Table 2). FNAC results of four patients showed benign features; however, after hemithyroidectomy, histology showed a PTC $(n=3)$ and FTC $(n=1)(30)$.

Based on histologic results, subsequent DTCs were two times more likely to be bilateral at diagnosis $(15 / 25$, $60 \%)$ than sporadic DTCs $(28 / 92,30 \%)(P=0.024)$ and tumors tended to be more often smaller in subsequent DTC patients (size at diagnosis $1.90(0.10-5.00) \mathrm{cm}$ vs $2.40(0.60-6.50) \mathrm{cm}(P=0.051))$. Of all, DTC tumors sized $<1 \mathrm{~cm}$ were more often seen in subsequent DTC patients $(6 / 23,26 \%)$ compared to sporadic DTC patients $(5 / 71$, $7 \%)(P=0.023)$. Conversely, in only $8.6 \%(2 / 23)$ of the subsequent DTC patients the tumor size was $\geq 4.0 \mathrm{~cm}$ vs $21.1 \%(15 / 71)$ of the sporadic DTC patients $(P=0.226)$ (Table 2). Approximately $15 \%$ of DTCs were follicular carcinomas, in both groups.

For evaluation of radiation effects specifically on the presentation of DTC, a subgroup analysis was done for the CCS with a history of neck radiation, TBI or MIBG treatment $(n=22)$. A significant difference was found in tumor size (median $1.25 \mathrm{~cm}$ vs $2.40 \mathrm{~cm}$, respectively
$(P=0.010))$. No difference was found in tumor size between CCS without radiation exposure and their matched controls (median tumor size $2.80 \mathrm{~cm}$ vs $2.40 \mathrm{~cm}$ respectively, $P=0.847)$.

In $15 / 23(65.2 \%)$ and $37 / 80(46.3 \%)$ of subsequent and sporadic DTC patients, respectively, the tumor had a multifocal character $(P=0.109)$. No significant differences were found in spread of DTC with regards to encapsulation of the tumor $(P=0.177)$, tumor capsular invasion $(P=0.186)$, extracapsular growth $(P=0.743)$, extra thyroid extension $(P=0.386)$, vessel invasion $(P=0.467)$ or lymph-node metastases $(P=0.277)$. Subsequent DTC patients showed more frequent $\mathrm{T} 1 \mathrm{a}$ staging compared to sporadic DTC patients $(P=0.046)$. Overall, TNM stage did not differ between subsequent DTC patients and sporadic DTC patients $(P=0.701)$.

Thirteen out of 21 (61.9\%) of the subsequent DTC patients had lymph-node metastasis vs 41/77 (53.2\%) in the sporadic patients. Of the six subsequent DTC patients with papillary microcarcinoma $(<1 \mathrm{~cm})$, two had lymphnode metastases. No association was found between tumor size and occurrence of lymph-node metastases. In total, seven patients had distant (lung) metastases, of which six were $\leq 18$ years at diagnosis. The tumor size of these patients ranged between 1.0 and $6.2 \mathrm{~cm}$. In both groups, the prevalence of lung metastases were similar, $7.7 \%$ vs $7.1 \%$ in subsequent vs sporadic DTC patients.

DTC treatment None of the surgical treatment characteristics differed between subsequent vs sporadic patients. All patients $(n=124)$ underwent one or more surgical procedures as part of their DTC treatment. Total thyroidectomy, unilateral hemithyroidectomy, and bilateral hemithyroidectomy were performed in, respectively, $66.7 \%, 7.4 \%$, and $25.9 \%$ of the subsequent DTC patients and in $55.9 \%, 1.1 \%$ and $43.0 \%$ of the sporadic DTC patients $(P=0.060)$ (Table 3$)$. Fifty-two percent and $52.7 \%$ of the subsequent and sporadic DTC patients, respectively, underwent lymph-node dissection(s) of central and/or lateral levels, and I-131 treatment was administered in $85.2 \%$ and $98.9 \%$ of, respectively, subsequent DTC vs sporadic DTC patients. A significant difference was found between the number of DTC patients that were treated with I-131 $(23 / 27,85.2 \%$ vs $92 / 93,98.9 \%, P=0.009)$. Three out of four subsequent DTC patients who did not receive I-131 treatment had been diagnosed with a papillary microcarcinoma with no lymph node metastases, one patient had undergone total thyroidectomy at time of data collection and may have been given RAI at later time during follow-up. 
Table 1 Comparison of presentation between subsequent DTC patients and sporadic DTC patients in The Netherlands (1968-2015).

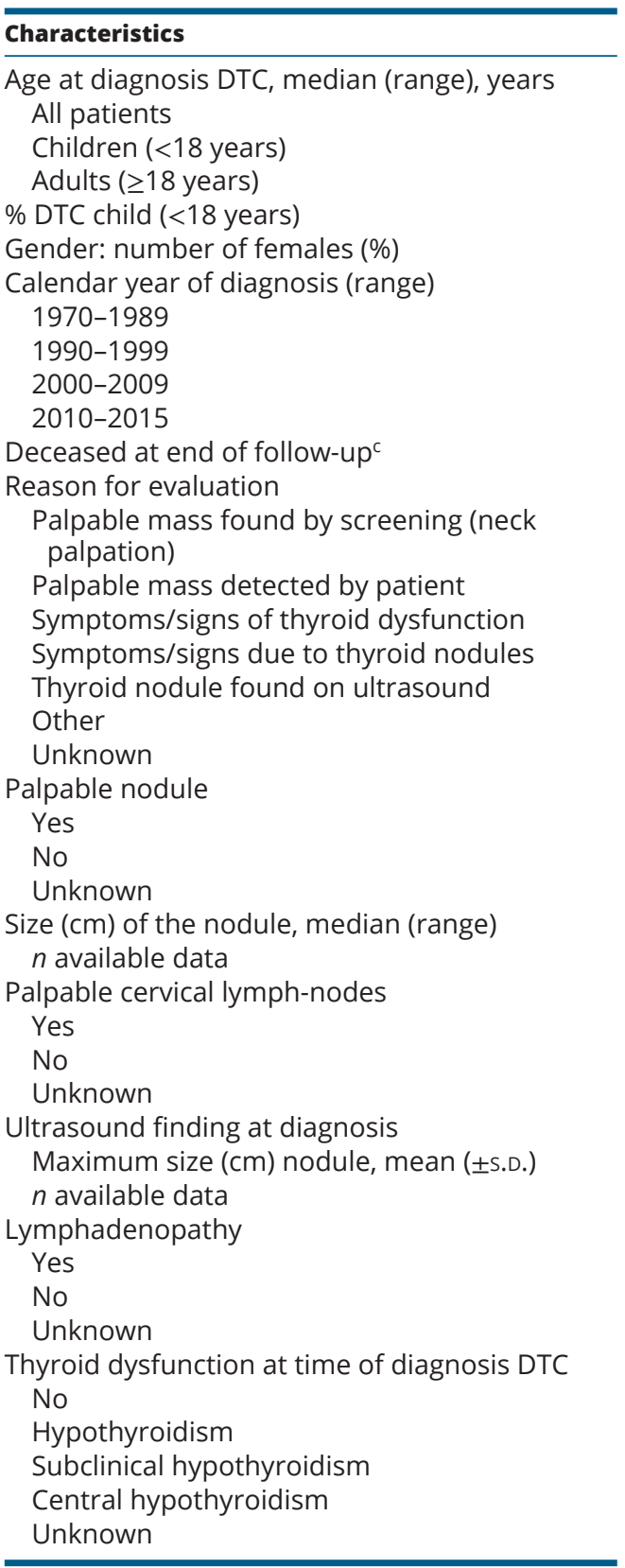

\begin{tabular}{c}
\hline Subsequent DTC $(n=31)$ \\
$25.6(6.1-38.0)$ \\
$13.3(6.1-17.7)$ \\
$29.8(18.03-38.0)$ \\
$7(23 \%)$ \\
$21(67.7 \%)$ \\
$1986-2015$ \\
$1(3.2 \%)$ \\
$5(16.1 \%)$ \\
$11(35.5 \%)$ \\
$14(45.2 \%)$
\end{tabular}

$4(13 \%)$

$12(46.2 \%)$

$9(34.6 \%)$

$2(7.7 \%)$

$0(0 \%)$

$0(0 \%)$

$3(11.5 \%)$

5

$21(87.5 \%)$

$3(12.5 \%)$

7

$3.5(0.5-5.0)$

$12 / 31$

$6(33.3 \%)$

$12(66.7 \%)$

13

$2.3(1.2)$

$18 / 31$

$5(38.5 \%)$

$8(61.5 \%)$

18

$12(70.6 \%)$

1 (5.9\%)

$3(17.6 \%)$

1 (5.9\%)

14

\begin{tabular}{|c|c|}
\hline Sporadic DTC ${ }^{(}(n=93)$ & $P$-value ${ }^{b}$ \\
\hline $26.3(5.8-38.7)$ & 0.875 \\
\hline $13.5(5.8-17.7)$ & 0.797 \\
\hline 29.3(18.2-38.7) & 0.982 \\
\hline \multicolumn{2}{|l|}{$20(22 \%)$} \\
\hline \multicolumn{2}{|l|}{$67(72.0 \%)$} \\
\hline \multicolumn{2}{|l|}{$1973-2015$} \\
\hline \multicolumn{2}{|l|}{$12(13.0 \%)$} \\
\hline \multicolumn{2}{|l|}{$14(15.1 \%)$} \\
\hline $44(47.3 \%)$ & 0.236 \\
\hline \multicolumn{2}{|l|}{$23(24.7 \%)$} \\
\hline $0(0 \%)$ & $0.003^{*}$ \\
\hline $4(8.3 \%)$ & 0.001 * \\
\hline \multicolumn{2}{|l|}{$33(68.8 \%)$} \\
\hline \multicolumn{2}{|l|}{$2(4.2 \%)$} \\
\hline \multicolumn{2}{|l|}{$4(8.3 \%)$} \\
\hline \multicolumn{2}{|l|}{$1(2.1 \%)$} \\
\hline \multicolumn{2}{|l|}{$4(8.3 \%)$} \\
\hline \multicolumn{2}{|l|}{45} \\
\hline & NA \\
\hline \multicolumn{2}{|l|}{$38(92.7 \%)$} \\
\hline \multicolumn{2}{|l|}{$3(7.3 \%)$} \\
\hline \multicolumn{2}{|l|}{52} \\
\hline \multirow{3}{*}{$\begin{array}{c}3.0(1.0-6.0) \\
24 / 93\end{array}$} & NA \\
\hline & \\
\hline & NA \\
\hline \multicolumn{2}{|l|}{$12(37.5 \%)$} \\
\hline \multicolumn{2}{|l|}{$20(62.5 \%)$} \\
\hline \multicolumn{2}{|l|}{61} \\
\hline & NA \\
\hline \multicolumn{2}{|l|}{$2.6(0.9)$} \\
\hline \multicolumn{2}{|l|}{$14 / 93$} \\
\hline & NA \\
\hline \multicolumn{2}{|l|}{5 (50.0\%) } \\
\hline \multicolumn{2}{|l|}{$5(50.0 \%)$} \\
\hline \multicolumn{2}{|l|}{83} \\
\hline & NA \\
\hline \multicolumn{2}{|l|}{15 (88.2\%) } \\
\hline \multicolumn{2}{|l|}{$2(11.8 \%)$} \\
\hline \multicolumn{2}{|l|}{$0(0 \%)$} \\
\hline $0(0 \%)$ & \\
\hline 76 & \\
\hline
\end{tabular}

Percentages of known variables are shown, $P$-value* significant $<0.05$; ${ }^{\mathrm{A} A l l}$ control patients were matched by age at diagnosis, gender, and calendar year of diagnosis; bMissing or unknown values are excluded from statistical testing. For characteristics with $>50 \%$ missing values per group, $P$-values were not calculated (denoted as NA, Not Applicable); 'Causes of death: due to other malignancies $(n=3)$ or non-cancer related death $(n=1)$.

DTC, differentiated thyroid carcinoma.

Disease recurrence and disease status at last moment of follow-up Median follow-up time after DTC diagnosis was 5.2 years $(0.1-22.5)$ for subsequent DTC vs 7.5 years (0.7-41.5) for sporadic DTC patients (Table 3). Recurrence rate between the two groups was comparable; $4 / 20(20.0 \%)$ of the subsequent DTC and in 10/88 (11.4\%) of the sporadic DTC patients $(P=0.288)$ (have) had recurrent disease.
At last moment of follow-up, the disease status was found to be different $(P=0.024)$ in the four categories, with remission of disease in $18 / 23$ (78.3\%) and 78/84 (92.9\%) of subsequent and sporadic DTC patients, respectively. Persistent disease was similar $(4.3 \%$ and $4.8 \%$, respectively). In both groups, two patients experienced recurrence of disease at last moment of follow-up. Outcome 
Table 2 Comparison of cytology and histology between subsequent DTC patients and sporadic DTC patients in The Netherlands (1968-2015).

\section{Cytology}

Mean number of FNACs ${ }^{b}$

$n$ available data

FNAC findings ${ }^{c}$

Non diagnostic

Malignant

Indeterminate

Benign

Unknown

Histology DTC

Papillary thyroid carcinoma

Papillary microcarcinoma $(<1 \mathrm{~cm})$

Follicular thyroid carcinoma

Unknown

Papillary (micro)carcinoma

Classic variant

Follicular

Diffuse sclerosing

Other

Unknown

Follicular carcinoma

Minimal invasive

Widely invasive

Unknown

DTC Laterality

Unilateral

Bilateral

Isthmus

Unknown

Multifocality

Yes

No

Unknown

Tumor size (cm; largest tumor nodule) median (range)

$n$ available data

Tumor size categories ${ }^{d}$

$$
\begin{aligned}
& 0.1-0.9 \mathrm{~cm} \\
& 1.0-1.9 \mathrm{~cm} \\
& 2.0-2.9 \mathrm{~cm} \\
& 3.0-3.9 \mathrm{~cm} \\
& 4.0-4.9 \mathrm{~cm} \\
& >5.0 \mathrm{~cm}
\end{aligned}
$$

Histology - Spread of DTC

Encapsulated

Yes

No

Unknown

Tumor capsular invasion

Yes

No

Unknown

Extracapsular growth

Yes

No

Unknown

\begin{tabular}{c}
\hline Subsequent $(n=$ \\
\hline 1.55 \\
$22 / 31$ \\
$2(13.3 \%)$ \\
$6(40.0 \%)$ \\
$5(33.3 \%)$ \\
$2(13.3 \%)$ \\
16
\end{tabular}

$16(61.5 \%)$

$6(23.1 \%)$

$4(15.4 \%)$

5

$10(45.5 \%)$

$10(45.5 \%)$

$1(4.5 \%)$

$1(4.5 \%)$

0

$3(100 \%)$

$0(0 \%)$

1

$10(40.0 \%)$

$15(60.0 \%)$

$0(0 \%)$

6

$15(65.2 \%)$

$8(34.8 \%)$

8

$1.90(0.10-5.00)$

$23 / 31$

$6(26.1 \%)$

$6(26.1 \%)$

$5(21.7 \%)$

$4(17.4 \%)$

1 (4.3\%)

$1(4.3 \%)$

$14(73.7 \%)$

$5(26.3 \%)$

12

$10(71.4 \%)$

$4(28.6 \%)$

0

$7(58.3 \%)$

$5(41.7 \%)$

2

\begin{tabular}{c}
\hline Sporadic $(n=93)$ \\
\hline 1.44 \\
$39 / 93$ \\
\\
$4(13.8 \%)$ \\
$15(51.7 \%)$ \\
$8(27.6 \%)$ \\
$2(6.9 \%)$ \\
64
\end{tabular}

$72(79.1 \%)$

$7(7.5 \%)$

$14(15.4 \%)$

0

$$
\begin{gathered}
28(50.0 \%) \\
20(35.7 \%) \\
2(3.6 \%) \\
6(10.7 \%) \\
23 \\
7(87.5 \%) \\
1(12.5 \%) \\
6
\end{gathered}
$$

$60(65.2 \%)$

$28(30.4 \%)$

$4(4.3 \%)$

1

$37(46.3 \%)$

$43(53.8 \%)$

13

$2.40(0.60-6.50)$

$71 / 93$

$5(7.0)$

$17(23.9 \%)$

$23(32.4 \%)$

$11(15.5 \%)$

$8(11.3 \%)$

$7(9.9 \%)$

$59(86.8 \%)$

$9(13.2 \%)$

25

$29(51.8 \%)$

$27(48.2 \%)$

3

$19(34.5 \%)$

$36(65.5 \%)$

4
0.095

0.051

NA

0.233

1.000

$0.024 *$

0.109

0.239

0.177

0.186

0.743

(Continued) 
Table 2 Continued.

\begin{tabular}{l}
\hline Cytology \\
\hline Extrathyroid extension (tissue invasion) \\
Yes \\
No \\
Unknown \\
Vessel invasion \\
Yes \\
No \\
Unknown \\
Lymph-node metastases \\
Yes \\
No \\
Unknown \\
TNM classification 7th editione \\
T \\
T1 \\
T2 \\
T3 \\
T4 \\
Tx \\
N \\
N0 \\
N1a-N1 \\
Nx \\
M \\
M0 \\
M1 \\
Mx
\end{tabular}

\begin{tabular}{cc}
\hline Subsequent $(n=31)$ \\
\hline $26(33.3 \%)$ \\
$12(66.7 \%)$ \\
\\
$5(26.3 \%)$ \\
$14(73.7 \%)$ \\
12 \\
$13(68.4 \%)$ \\
$6(31.6 \%)$ \\
12
\end{tabular}

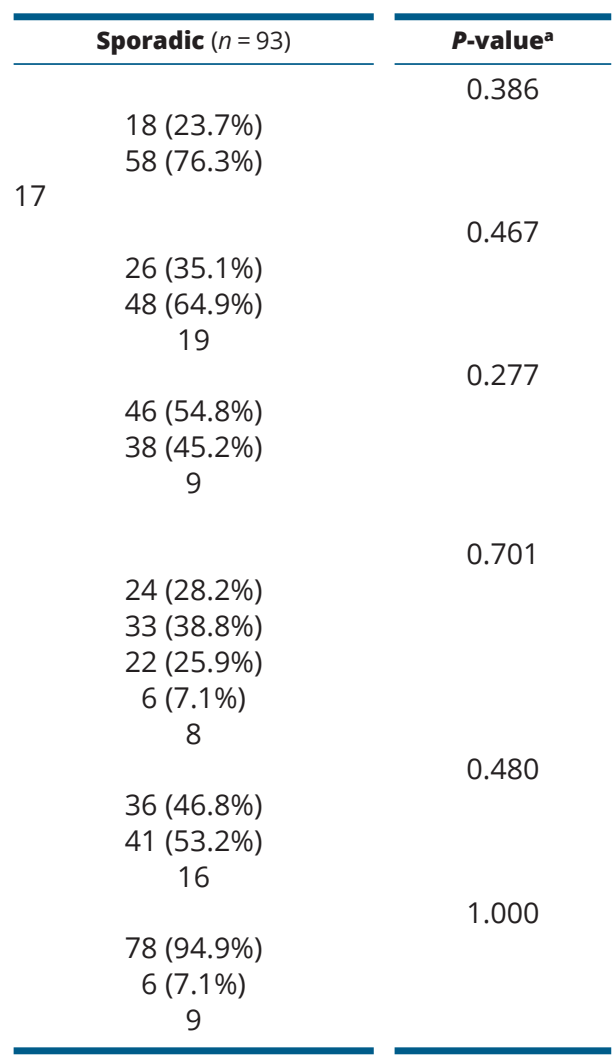

Percentages of known variables are shown, $P$-value* significant $<0.05$; ${ }^{a}$ Missing or unknown values were excluded from statistical testing; ${ }^{b}$ Mean number of all performed FNACs before diagnosis DTC; 'Last FNAC before histological diagnosis $<6$ months; ${ }^{\mathrm{d}}$ Tumor categories are based on continuous data. In two sporadic DTC patients, the pathology report showed microcarcinoma; however, no exact tumor size was mentioned; eThe $7^{\text {th }}$ edition of TNM classification was used for all patients, if previous editions were used in the patient record, all were recoded into the $7^{\text {th }}$ edition of TNM classification; ${ }^{\mathrm{f}} \mathrm{M} 1$ = only lung metastases were found.

FNAC, fine needle aspiration cytology.

could not be assessed for two subsequent DTC patients, because diagnosis of DTC was $<1$ year ago. Results did not change when excluding pediatric DTC patients and microcarcinomas, except for disease status at last moment of follow-up (Supplementary Table A, see section on supplementary materials given at the end of this article).

Three out of 14 (21.4\%) and 10/88 (11.4\%) of subsequent and sporadic DTC patients, respectively, had a history of positive serum $\mathrm{Tg}$ antibodies $(P=0.381)$. Vital status at end of follow-up differed between the groups: four subsequent DTC patients were deceased at end of follow-up due to other malignancies $(n=3)$ or non cancer related death $(n=1)$, whereas sporadic DTC patients were all alive at last moment of follow-up. The distributions of age, gender, and diagnosis period were fairly comparable as expected from the per-protocol matching.

Surgical complications Documented transient hypoparathyroidism and documented permanent hypopara- thyroidism were observed in, respectively, 4/31 (12.9\%) and $11 / 31(35.5 \%)$ of the subsequent DTC patients and in $21 / 93(22.6 \%)$ and $33 / 93(35.5 \%)$ of the sporadic DTC patients $(P=0.456)$. Documented postoperative transient and permanent RLN injury was found in, respectively, $1 / 31(3.2 \%)$ and $3 / 31(9.7 \%)$ of the subsequent DTC patients and in 7/93 (7.5\%) and 17/93 (18.3\%) of the sporadic DTC patientens $(P=0.327)$.

\section{Discussion}

Radiation therapy including the neck region for childhood cancer may result in DTC. It has been suggested that subsequent DTC in CCS may also be related to chemotherapy $(2,3,4,6)$. In order to counsel CCS on the most appropriate way to screen for DTC, more knowledge is required upon its behavior in comparison to sporadic thyroid cancer. The prognosis of sporadic thyroid cancer is known to be excellent, even when found in advanced 
Table 3 Comparison of treatment, outcome, and complication rates between subsequent DTC patients and sporadic DTC patients in The Netherlands (1968-2015).

\begin{tabular}{|c|c|c|c|}
\hline & Subsequent $(n=31)$ & Sporadic $(n=93)$ & P-value ${ }^{a}$ \\
\hline \multicolumn{4}{|l|}{ Initial treatment of thyroid cancer } \\
\hline Surgical treatment & & & 0.060 \\
\hline Total thyroidectomy & $18(66.7 \%)$ & $52(55.9 \%)$ & \\
\hline Unilateral hemithyroidectomy & $2(7.4 \%)$ & $1(1.1 \%)$ & \\
\hline Bilateral hemithyroidectomy ${ }^{b}$ & $7(25.9 \%)$ & $40(43.0 \%)$ & \\
\hline Unknown & 4 & 0 & \\
\hline Lymph-node dissection (LND) & & & 0.732 \\
\hline None & $12(48.0 \%)$ & $43(47.3 \%)$ & \\
\hline Central LND & $2(8.0 \%)$ & $11(12.1 \%)$ & \\
\hline LND incl. lateral levels & $9(36.0 \%)$ & $34(37.4 \%)$ & \\
\hline LND, location unknown & $2(8.0 \%)$ & $3(3.3 \%)$ & \\
\hline Unknown & 6 & 2 & \\
\hline \multicolumn{4}{|l|}{$\mathrm{I}-131$ treatment $^{\mathrm{c}}$} \\
\hline Number of patients treated with I-131 treatment & $23 / 27(85.2 \%)$ & 92/93 (98.9\%) & 0.009 \\
\hline Number of I-131 treatments, median (range) & $1(1-3)$ & $2(1-6)$ & 0.268 \\
\hline $\begin{array}{l}\text { Cumulative administered dose of I-131 } \\
\text { treatment, median (range) GBq }\end{array}$ & $5.399(1.850-17.910)$ & $7.400(1.480-35.150)$ & 0.242 \\
\hline \multicolumn{4}{|l|}{ Recurrence } \\
\hline Recurrence & & & 0.288 \\
\hline Yes & $4(20.0 \%)$ & $10(11.4 \%)$ & \\
\hline No & $16(80.0 \%)$ & $78(88.6 \%)$ & \\
\hline Unknown & 11 & 5 & \\
\hline \multicolumn{4}{|l|}{ Treatment recurrence } \\
\hline Lymph-node dissection & $2(40.0 \%)$ & $3(33.3 \%)$ & \\
\hline I-131 treatments & $3(60.0 \%)$ & $6(66.7 \%)$ & \\
\hline $\begin{array}{l}\text { Cumulative administered dose of I-131 } \\
\text { treatment, median (range) GBq }\end{array}$ & $5.550(5.550-6.008)$ & $5.550(5.550-5.550)$ & \\
\hline Most recent Tg determination elevated & & & 0.166 \\
\hline Yes & $3(14.3 \%)$ & $5(5.4 \%)$ & \\
\hline No & $18(85.7 \%)$ & $87(94.6 \%)$ & \\
\hline Unknown & 10 & 1 & \\
\hline \multicolumn{4}{|l|}{ Disease status at last moment of follow-up } \\
\hline $\begin{array}{l}\text { Time (years) between Dx and last moment of } \\
\text { follow-up, median (range) }\end{array}$ & $5.2(0.1-22.5)$ & $7.5(0.7-41.5)$ & 0.240 \\
\hline$n$ available data & $23 / 31$ & $92 / 93$ & \\
\hline Disease status at last moment of follow-up ${ }^{d}$ & & & $0.024 *$ \\
\hline Remission & $18(78.3 \%)$ & $78(92.9 \%)$ & \\
\hline Active disease: persistent disease & $1(4.3 \%)$ & $4(4.8 \%)$ & \\
\hline Active disease: recurrence & $2(8.7 \%)$ & $2(2.4 \%)$ & \\
\hline Last treatment $<1$ year ago & $2(8.7 \%)$ & $0(0 \%)$ & \\
\hline Unknown & 8 & 9 & \\
\hline T4 supplementation & & & 0.216 \\
\hline Yes & $24(96.0 \%)$ & $91(100 \%)$ & \\
\hline No & $1(4.0 \%)$ & $0(0 \%)$ & \\
\hline Unknown & 6 & 2 & \\
\hline Serum Tg antibodies & & & 0.381 \\
\hline Yes & $3(21.4 \%)$ & $10(11.4 \%)$ & \\
\hline No & $11(78.6 \%)$ & $78(88.6 \%)$ & \\
\hline Unknown & 17 & 5 & \\
\hline \multicolumn{4}{|l|}{ Surgical Complications } \\
\hline Documented hypoparathyroidism & & & 0.456 \\
\hline Transient hypoparathyroidism & $4(12.9 \%)$ & $21(22.6 \%)$ & \\
\hline Permanent hypoparathyroidism & $11(35.5 \%)$ & $33(35.5 \%)$ & \\
\hline Documented recurrent laryngeal nerve (RLN) injury & & & 0.327 \\
\hline Transient RLN injury & $1(3.2 \%)$ & $7(7.5 \%)$ & \\
\hline Permanent RLN injury & $3(9.7 \%)$ & $17(18.3 \%)$ & \\
\hline
\end{tabular}

Percentages of known variables are shown, $P$-value* significant $<0.05$; ${ }^{a}$ Missing or unknown values excluded from statistical testing; ${ }^{b}$ Consecutive

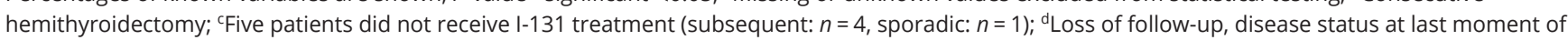
follow-up unknown in 17 patients in total (subsequent $n=8$, sporadic $n=9$ ). 
stage. However, the behavior of radiation-induced DTC has been studied insufficiently, for which this matched cohort analysis was performed.

The unique data in this study, integrated from three national initiatives spanning four decades of inclusion, enabled us to address the mode of detection and presentation of subsequent DTC. Our results demonstrate that CCS with subsequent DTC more likely tend to present with smaller tumors and bilateral disease than patients with sporadic DTC. Other characteristics were statistically similar. Of note, one-third of the patients with subsequent DTC did not have a history of radiotherapy directed to the head/neck/upper chest.

A noteworthy finding of this study is that the number of small tumors was significantly increased in the subsequent DTC group, especially in CCS with a history of neck radiation, TBI or MIBG, compared to sporadic patients. These results are in agreement with several previous studies $(14,15,18,20,31)$ and might be explained by the fact that CCS are carefully followed at follow-up clinics, leading to the detection of DTC in an earlier $\mathrm{T}$ stage. Tumor size has shown to be an important factor influencing DTC prognosis and we confirmed from previous findings that tumor size is not associated with the occurrence of lymph-node metastases $(32,33)$.

The high prevalence of microcarcinoma among subsequent DTC patients did not result in improved outcome results, such as decreased recurrence rates or surgical complications when compared to sporadic DTC. However, it was remarkable that three subsequent DTC patients with microcarcinoma had not been treated with I-131, while all patients with sporadic patients and micorcarcinoma $(n=7)$ had been treated with I-131. This may reflect more hesitance in providers to further expose cancer survivors to I-131.

Bilateral tumors were significantly more often diagnosed in subsequent DTC patients. This is consistent with the hypothesis that radiation exposure results in diffuse toxicity and is the major contributing factor in DTC etiology.

Our data confirms previous data that multifocal tumors are more frequent in subsequent DTC patients compared to sporadic DTC patients $(19,23)$. In the study by Rubino et al., multifocality was more frequent in those who received higher radiation dose at younger age suggesting that multifocality is a direct consequence of radiation exposure (19).

Bilaterality requires total thyroidectomy, and it was observed that, in this cohort, indeed only $1 \%$ of patients with subsequent DTC were treated with hemithyroidecomy, in comparison to $7 \%$ of sporadic DTC.

Next to bilaterality, multifocality and tumor size, no other significant differences were found in this study between subsequent and sporadic DTC patients regarding histological findings.

In this cohort, we found a striking high incidence of documented permanent hypoparathyroidism in all DTC patients, when compared to previous studies in children and adults $(34,35,36,37)$. These high percentages should be further explored and may possibly be explained by the strict definition of hypoparathyroidism used in our study. To reduce complications of treatment and considering the rareness of the disease, care for DTC should be centralized and only be done in an experienced DTC center.

Recurrence rates were in line with previous literature, and neither differences in recurrence rates between groups were found, nor was there mortality due to DTC in both groups $(38,39)$.

At last moment of follow-up, patients with subsequent DTC had significantly more frequent persistent disease. A possible explanation may be the fact that, in the subsequent DTC patients, the last treatment was $<1$ year ago in two patients, in comparison to the sporadic DTC patients of whom last treatment was $>1$ year ago in all patients. This must be further studied in future cohorts.

One-third of the subsequent DTC patients in this study had not been exposed to radiotherapy. This implies that other causes for subsequent tumor formation must be considered which justifies future research. All had been treated with chemotherapy. Effects of alkylating agents and anthracyclines on the thyroid gland were demonstrated in a large pooling effort by Veiga et al.(2, $3)$. Also, genetic predisposing factors may increase risk of DTC among CCS (40). The large number of CT scans ( $>50$ for some individuals) is not a negligible factor and should be explored in subsequent work $(41,42)$.

The strength of this study is the fact that data were retrieved from well-characterized study populations including valid methods with retrospective and prospective case-finding for DTC. We estimate that our study captures $>90 \%$ of all patients with subsequent DTC in The Netherlands in the study period. By matching the CCS to patients with sporadic DTC, comparisons could be made unbiased by factors affecting tumor and outcome characteristics. Lastly, for a fair proportion of the DTC cases, in-depth data were available, making these comparisons possible.

A weakness of this study is the number of missing values owing to the character of the retrospective chart 
review. The missing data in most cases could be explained by the fact that these patients presented in non-academic hospitals and were then referred to academic hospitals. We were only able to retrieve data from patient charts in the academic hospitals and therefore data are missing. To give insight in the magnitude of missing data, percentages are based on the number of CCSs with available data in the denominator. The fact that subjects included in this study had not been treated with the same treatment protocol in a systematic way, because of pluralism in hospitaldependent treatment protocols, may also be considered a limitation.

Also, despite the fact that for patients with subsequent DTC this was a national cohort, the numbers were quite low, making multivariate or survival analysis not possible. Outcomes are therefore not controlled for tumor characteristics and treatment methods. In the future, international collaboration should be aimed to create larger cohorts enabling more solid analyses.

Despite the fact that we had nationwide coverage, our cohort is a modest size sample which precludes strong conclusions. There are several aspects that should be taken into account. Although no difference was found in overall TNM stage, more tumors $<1 \mathrm{~cm}$ were found (T1a staging) in subsequent DTC patients This may possibly be a consequence of surveillance (10). The results of this study cannot be used, however, to inform screening strategies, such as neck palpation or thyroid ultrasound. For the future, we recommend that all patients with DTC are prospectively recorded and treated in centers of expertise (12). In the medical files of these patients, data on diagnostics, treatment, adverse effects and outcome should be recorded according to standardized definitions to allow for future evaluation of care (12). Development for standardization of care for children with DTC in The Netherlands and in larger European consortia are underway. For patients $>18$ years of age, standards of care for adults may be used.

In conclusion, patients with subsequent DTC seem to present with smaller tumors and more frequent bilateral tumor localizations than patients with sporadic DTC. In terms of morbidity and mortality, subsequent DTC seems to be similar to that of sporadic DTC. The results of this study do not provide evidence or arguments that different or more aggressive treatment regimens should be used.

The multifocality and bilaterality of DTC after treatment for childhood cancer must be taken into account when deciding on the surgical procedure.
Differences in outcome and prognosis of DTC in CCS without following an active surveillance program could not be excluded.

These results are an important cornerstone for the further development of existing surveillance programs and treatment guidelines for CCS at risk for or presenting with DTC (11). Follow-up studies are needed to explore the potential cause of subsequent DTC in patients without radiation treatment for their primary cancer.

\section{Supplementary materials}

This is linked to the online version of the paper at https://doi.org/10.1530/ EJE-20-0153.

Declaration of interest

The authors have nothing to disclose.

\section{Funding}

The authors declare that there is no conflict of interest that could be perceived as prejudicing the impartiality of this study.

\section{Author contribution statement}

S C Clement, C A Lebbink, C M Ronckers and H M van Santen contributed equally to this work.

\section{Acknowledgements}

The DCOG LATER Study Group includes the following: LCM Kremer, J Loonen, E van Dulmen-den Broeder, WJE Tissing, MM van den Heuvel-Eibrink, M van der Heiden, AB Versluys, HJH van der Pal, D Bresters, S Neggers, FE van Leeuwen, G Janssens, J Maduro. The Dutch (Pediatric) Thyroid Cancer Consortium includes the following: G Bocca, JGM Burgerhof, EWCM van Dam, B Havekes, EPM Corssmit, RT Netea-Maier, RP Peeters, JWA Smit, JTM Plukker, and $\mathrm{AH}$ Brouwers.

\section{References}

1 Mariotto AB, Rowland JH, Yabroff KR, Scoppa S, Hachey M, Ries L $\&$ Feuer EJ. Long-term survivors of childhood cancers in the United States. Cancer Epidemiology, Biomarkers and Prevention 200918 1033-1040. (https://doi.org/10.1158/1055-9965.EPI-08-0988)

2 Veiga LHS, Lubin JH, Anderson H, de Vathaire F, Tucker M, Bhatti P, Schneider A, Johansson R, Inskip P, Kleinerman R et al. A pooled analysis of thyroid cancer incidence following radiotherapy for childhood cancer. Radiation Research 2012178 365-376. (https://doi. org/10.1667/rr2889.1)

3 Veiga LHS, Bhatti P, Ronckers CM, Sigurdson AJ, Stovall M, Smith SA, Weathers R, Leisenring W, Mertens AC, Hammond S et al. Chemotherapy and thyroid cancer risk: a report from the childhood cancer survivor study. Cancer Epidemiology, Biomarkers and Prevention 201221 92-101. (https://doi.org/10.1158/1055-9965.EPI-11-0576)

4 Bhatti P, Veiga LHS, Ronckers CM, Sigurdson AJ, Stovall M, Smith SA, Weathers R, Leisenring W, Mertens AC, Hammond S et al. Risk of 
second primary thyroid cancer after radiotherapy for a childhood cancer in a large cohort study: an update from the childhood cancer survivor study. Radiation Research 2010174 741-752. (https://doi. org/10.1667/RR2240.1)

5 Teepen JC, van Leeuwen FE, Tissing WJ, van Dulmen-den Broeder E, van den Heuvel-Eibrink MM, van der Pal HJ, Loonen JJ, Bresters D, Versluys B, Neggers SJCMM et al. Long-term risk of subsequent malignant neoplasms after treatment of childhood cancer in the DCOG LATER study cohort: role of chemotherapy. Journal of Clinical Oncology 201735 2288-2298. (https://doi.org/10.1200/ JCO.2016.71.6902)

6 Lubin JH, Adams MJ, Shore R, Holmberg E, Schneider AB, Hawkins MM, Robison LL, Inskip PD, Lundell M, Johansson R et al. Thyroid cancer following childhood low-dose radiation exposure: a pooled analysis of nine cohorts. Journal of Clinical Endocrinology and Metabolism 2017102 2575-2583. (https://doi.org/10.1210/jc.2016-3529)

7 Van Santen HM, Tytgat GAM, Van De Wetering MD, Van EckSmit BLF, Hopman SMJ, Van Der Steeg AF, Nieveen Van Dijkum EJM \& Van Trotsenburg ASP. Differentiated thyroid carcinoma after 131I-MIBG treatment for neuroblastoma during childhood: description of the first two cases. Thyroid 201222 643-646. (https:// doi.org/10.1089/thy.2011.0464)

8 van Leeuwen FE \& Ronckers CM. Anthracyclines and alkylating agents: new risk factors for breast cancer in childhood cancer survivors? Journal of Clinical Oncology 201634 891-894. (https://doi. org/10.1200/JCO.2015.65.0465)

9 Hogan AR, Zhuge Y, Perez EA, Koniaris LG, Lew JI \& Sola JE. Pediatric thyroid carcinoma: incidence and outcomes in 1753 patients. Journal of Surgical Research 2009156 167-172. (https://doi.org/10.1016/j. jss.2009.03.098)

10 Dutch Childhood Oncology Group. Richtlijn follow-up na kinderkanker meer dan 5 jaar na diagnose. Publicaties op Maat, Den Haag/Amsterdam, the Netherlands, 2010. https://www.skion.nl/ workspace/uploads/richtlijn_follow-up_na_kinderkanker_deel_1_1.pdf

11 Clement SC, Kremer LCM, Verburg FA, Simmons JH, Goldfarb M, Peeters RP, Alexander EK, Bardi E, Brignardello E, Constine LS et al. Balancing the benefits and harms of thyroid cancer surveillance in survivors of childhood, adolescent and young adult cancer: recommendations from the International Late Effects of Childhood Cancer Guideline Harmonization Group in collaboration with the PanCareSurFup Consortium. Cancer Treatment Reviews 201863 28-39. (https://doi.org/10.1016/j.ctrv.2017.11.005)

12 Francis GL, Waguespack SG, Bauer AJ, Angelos P, Benvenga S, Cerutti JM, Dinauer CA, Hamilton J, Hay ID, Luster M et al. Management guidelines for children with thyroid nodules and differentiated thyroid cancer. Thyroid 201525 716-759. (https://doi. org/10.1089/thy.2014.0460)

13 Haugen BR, Alexander EK, Bible KC, Doherty G, Mandel SJ, Nikiforov YE, Pacini F, Randolph G, Sawka A, Schlumberger M et al. American Thyroid Association management guidelines for adult patients with thyroid nodules and differentiated thyroid cancer. Thyroid 20152016 1-133. (https://doi.org/10.1089/thy.2015.0020)

14 Sassolas G, Hafdi-Nejjari Z, Casagranda L, Berger C, Bournaud C, Decaussin-Petrucci M, Berger N \& Borson-Chazot F. Thyroid cancers in children, adolescents, and young adults with and without a history of childhood exposure to therapeutic radiation for other cancers. Thyroid 201323 805-810. (https://doi.org/10.1089/thy.2011.0370)

15 Rumyantsev PO, Saenko VA, Ilyin AA, Stepanenko VF, Rumyantseva UV, Abrosimov AY, Lushnikov EF, Rogounovitch TI, Shibata Y, Mitsutake N et al. Radiation exposure does not significantly contribute to the risk of recurrence of Chernobyl thyroid cancer. Journal of Clinical Endocrinology and Metabolism 2011 96 385-393. (https://doi.org/10.1210/jc.2010-1634)

16 Spinelli C, Bertocchini A, Antonelli A \& Miccoli P. Surgical therapy of the thyroid papillary carcinoma in children: experience with
56 patients $\leq 16$ years old. Journal of Pediatric Surgery 200439 1500-1505. (https://doi.org/10.1016/j.jpedsurg.2004.06.016)

17 Gow KW, Lensing S, Hill DA, Krasin MJ, McCarville MB, Rai SN, Zacher M, Spunt SL, Strickland DK \& Hudson MM. Thyroid carcinoma presenting in childhood or after treatment of childhood malignancies: an institutional experience and review of the literature. Journal of Pediatric Surgery 200338 1574-1580. (https://doi. org/10.1016/s0022-3468(03)00563-3)

18 Furlan JC \& Rosen IB. Prognostic relevance of previous exposure to ionizing radiation in well-differentiated thyroid cancer. Langenbeck's Archives of Surgery 2004389 198-203. (https://doi.org/10.1007/ s00423-003-0424-0)

19 Rubino C, Cailleux AF, Abbas M, Diallo I, Shamsaldin A, Caillou B, De Vathaire F \& Schlumberger M. Characteristics of follicular cell-derived thyroid carcinomas occurring after external radiation exposure: results of a case control study nested in a cohort. Thyroid 200212 299-304. (https://doi.org/10.1089/10507250252949423)

20 Shirahige Y, Ito M, Ashizawa K, Motomura T, Yokoyama N, Namba H, Fukata S, Yokozawa T, Ishikawa N, Mimura T et al. Childhood thyroid cancer: comparison of Japan and Belarus. Endocrine Journal $1998 \mathbf{4 5}$ 203-209. (https://doi.org/10.1507/endocrj.45.203)

21 Pacini F, Vorontsova T, Demidchik EP, Molinaro E, Agate L, Romei C, Shavrova E, Cherstvoy ED, Ivashkevitch Y, Kuchinskaya E et al. PostChernobyl thyroid carcinoma in Belarus children and adolescents: comparison with naturally occurring thyroid carcinoma in Italy and France. Journal of Clinical Endocrinology and Metabolism 199782 3563-3569. (https://doi.org/10.1210/jcem.82.11.4367)

22 Samaan NA, Schultz PN, Ordonez NG, Hickey RC \& Johnston DA. A comparison of thyroid carcinoma in those who have and have not had head and neck irradiation in childhood. Journal of Clinical Endocrinology and Metabolism 198764 219-223. (https://doi. org/10.1210/jcem-64-2-219)

23 Roudebush CP, Asteris GT \& DeGroot LJ. Natural history of radiationassociated thyroid cancer. Archives of Internal Medicine 1978138 1631-1634.

24 Keegan THM, Bleyer A, Rosenberg AS, Li Q \& Goldfarb M. Second primary malignant neoplasms and survival in adolescent and young adult cancer survivors. JAMA Oncology 20173 1554-1557. (https:// doi.org/10.1001/jamaoncol.2017.0465)

25 Teepen JC, Kok JL, van Leeuwen FE, Tissing WJE, Dolsma WV, van der Pal HJ, Loonen JJ, Bresters D, Versluys B, van den HeuvelEibrink MM et al. Colorectal adenomas and cancers after childhood cancer treatment: a DCOG-LATER record linkage study. Journal of the National Cancer Institute 2018110 758-767. (https://doi.org/10.1093/ jnci/djx266)

26 Klein Hesselink MS, Nies M, Bocca G, Brouwers AH, Burgerhof JGM, van Dam EWCM, Havekes B, van den Heuvel-Eibrink MM, Corssmit EPM, Kremer LCM et al. Pediatric differentiated thyroid carcinoma in the Netherlands: a nationwide follow-up study. Journal of Clinical Endocrinology and Metabolism 2016101 2031-2039. (https://doi.org/10.1210/jc.2015-3290)

27 Links TP, van Tol KM, Jager PL, Plukker JTM, Piers DA, Boezen HM, Dullaart RPF, de Vries EGE \& Sluiter WJ. Life expectancy in differentiated thyroid cancer: a novel approach to survival analysis. Endocrine-Related Cancer 200512 273-280. (https://doi.org/10.1677/ erc.1.00892)

28 Sobin LH, Gospodarowicz MK \& Wittekind Ch (eds). International Union Against Cancer (UICC) TNM Classification of Malignant Tumors, 7th ed. Oxford, UK: Wiley-Blackwell, 2009.

29 Clement SC, van Eck-Smit BLF, van Trotsenburg ASP, Kremer LCM, Tytgat GAM \& van Santen HM. Long-term follow-up of the thyroid gland after treatment with 131I-metaiodobenzylguanidine in children with neuroblastoma: importance of continuous surveillance. Pediatric Blood and Cancer 201360 1833-1838. (https:// doi.org/10.1002/pbc.24681) 
30 Li J, Wang Q, Wang L, Wang J, Wang D, Xin Z, Liu Y \& Zhao Q. Diagnostic value of fine-needle aspiration combined with ultrasound for thyroid cancer. Oncology Letters 201918 2316-2321. (https://doi. org/10.3892/ol.2019.10584). (available at: https://www.ncbi.nlm.nih. gov/pubmed/31402937)

31 Goldfarb M \& Freyer DR. Comparison of secondary and primary thyroid cancer in adolescents and young adults. Cancer 2014120 1155-1161. (https://doi.org/10.1002/cncr.28463)

32 Jin S, Bao W, Yang YT, Bai T \& Bai Y. Establishing a prediction model for lateral neck lymph node metastasis in patients with papillary thyroid carcinoma. Scientific Reports 20188 17355. (https://doi. org/10.1038/s41598-018-35551-9)

33 Machens A, Holzhausen HJ \& Dralle H. The prognostic value of primary tumor size in papillary and follicular thyroid carcinoma. Cancer 2005103 2269-2273. (https://doi.org/10.1002/cncr.21055)

34 Thomusch O, Machens A, Sekulla C, Ukkat J, Brauckhoff M \& Dralle $\mathrm{H}$. The impact of surgical technique on postoperative hypoparathyroidism in bilateral thyroid surgery: a multivariate analysis of 5846 consecutive patients. Surgery 2003133 180-185. (https://doi.org/10.1067/msy.2003.61)

35 Hundahl SA, Cady B, Cunningham MP, Mazzaferri E, McKee RF, Rosai J, Shah JP, Fremgen AM, Stewart AK \& Hölzer S. Initial results from a prospective cohort study of 5583 cases of thyroid carcinoma treated in the United States during 1996. Cancer 200089 202-217. (https://doi.org/10.1002/1097-0142(20000701)89:1<202::aidcncr27>3.0.co;2-a)

36 Haveman JW, van Tol KM, Rouwé CW, Piers DA \& Plukker JTM. Surgical experience in children with differentiated thyroid carcinoma. Annals of Surgical Oncology 200310 15-20. (https://doi. org/10.1245/aso.2003.03.002)
37 Thompson GB \& Hay ID. Current strategies for surgical management and adjuvant treatment of childhood papillary thyroid carcinoma. World Journal of Surgery 200428 1187-1198. (https://doi.org/10.1007/ s00268-004-7605-z)

38 Reiners C, Biko J, Haenscheid H, Hebestreit H, Kirinjuk S, Baranowski O, Marlowe RJ, Demidchik E, Drozd V \& Demidchik Y. Twenty-five years after Chernobyl: outcome of radioiodine treatment in children and adolescents with very high-risk radiation-induced differentiated thyroid carcinoma. Journal of Clinical Endocrinology and Metabolism 201398 3039-3048. (https://doi.org/10.1210/jc.20131059)

39 Rachmiel M, Charron M, Gupta A, Hamilton J, Wherrett D, Forte V $\&$ Daneman D. Evidence-based review of treatment and follow up of pediatric patients with differentiated thyroid carcinoma. Journal of Pediatric Endocrinology and Metabolism 200619 1377-1393. (https:// doi.org/10.1515/jpem.2006.19.12.1377)

40 Lindor NM, McMaster ML, Lindor CJ \& Greene MH. Concise handbook of familial cancer susceptibility syndromes - second edition. Journal of the National Cancer Institute 200838 1-93.

41 Su YP, Niu HW, Chen JB, Fu YH, Xiao GB \& Sun QF. Radiation dose in the thyroid and the thyroid cancer risk attributable to CT scans for pediatric patients in one general hospital of China. International Journal of Environmental Research and Public Health 201411 2793-2803. (https://doi.org/10.3390/ijerph110302793)

42 Meulepas JM, Ronckers CM, Smets AMJB, Nievelstein RAJ, Gradowska P, Lee C, Jahnen A, van Straten M, de Wit MY, Zonnenberg B et al. Radiation exposure from pediatric CT scans and subsequent cancer risk in the Netherlands. Journal of the National Cancer Institute 2019111 256-263. (https://doi.org/10.1093/jnci/ djy104)

Received 24 February 2020

Revised version received 8 April 2020

Accepted 20 May 2020 